\title{
A Bioinspired Threonine-based Antifreeze Protein Mimic with Potent Ice Recrystallization Inhibition Activity
}

Elizabeth A. Deleskya, Luis F. Garciab, Aparna J. Lobo ${ }^{c}$, Jaqueline D. Wallat ${ }^{d}$, Garret M. Miyake $^{b}$, and Wil V. Srubar III ${ }^{a, d, *}$

${ }^{\mathrm{a}}$ Materials Science and Engineering Program, ${ }^{\mathrm{C}}$ Department of Chemical and Biological Engineering, ${ }^{d}$ Department of Civil, Environmental, and Architectural Engineering, University of Colorado Boulder, ECOT 441 UCB 428, Boulder, Colorado 80309-0428 USA, bDepartment of Chemistry, Colorado State University, Fort Collins, CO 80523, USA; "Corresponding Author, T +1 303492 2621, F+1 303492 7317, E wsrubar@colorado.edu

Keywords: biomimicry, ice recrystallization inhibition, antifreeze proteins, ice-binding proteins

Ice growth mitigation is a pervasive challenge for multiple industries. In nature, ice-binding proteins (IBPs) demonstrate potent ice-growth prevention through ice recrystallization inhibition (IRI). However, IBPs are expensive, difficult to produce in large quantities, and exhibit minimal resilience to non-physiological environmental stressors, such as elevated $\mathrm{pH}$. For these reasons, researchers have turned to polymeric bioinspired mimics. To-date, however, no mimic has rivaled the ability of native IBPs to display IRI activity at ultra-low nanomolar concentrations. In this work, we study the IRI activity of peptides and polypeptides inspired by common ice-binding residues of IBPs to inform the synthesis and characterization of a potent bioinspired polymer mimic. We show first that the threonine polypeptide (pThr) displays the best IRI activity in 
phosphate-buffered saline (PBS). Second, we use $p T h r$ as a molecular model to synthesize and test a new bioinspired polymer mimic, poly(2-hydroxypropyl methacrylamide) ( $p H P M A)$. We show that $p H P M A$ exhibits potent IRI activity in neutral PBS at nanomolar concentrations. These results substantiate that $P H P M A$ outperforms poly(vinyl alcohol) (PVA), the current top performing IBP mimic in the field, in terms of effectiveness at mitigating ice crystal growth at concentrations akin to native IBPs.

\section{Introduction}

Water is one of the only substances on earth that expands when it freezes. A unit volume of water can expand by $9 \%$ upon crystallization, ${ }^{1,2}$ which can cause damage to a multitude of watercontaining materials and systems. For example, ice expansion during cryopreservation can damage proteins or rupture cells. ${ }^{3-6}$ In food preservation, the growth of ice crystallites can alter the texture of foods, rendering them unpalatable. ${ }^{7.8}$ In infrastructure, the cyclic freezing and thawing of water within concrete can induce cracking and failure. ${ }^{9-11}$ Methods of preventing ice growth and subsequent damage can extend the service life of materials, which can save money, resources, and time in the process.

In nature, a multitude of organisms have evolved to solve the ice expansion problem. Ice-binding proteins (IBPs) are a diverse category of proteins that have emerged through convergent evolution among different organisms, including plants, ${ }^{12,13}$ fungi, ${ }^{12,14,15}$ fish, ${ }^{16-18}$ insects, ${ }^{19,20}$ and microorganisms..$^{12,21,22}$ Although IBPs display a variety of structures, genomic sequences, and icebinding residues, ${ }^{23,24}$ IBPs demonstrate a similar function: preventing ice crystal growth. The prevailing mechanism by which IBPs inhibit ice growth postulates that ice-interaction is facilitated by quasi-liquid anchored clathrate-waters at the ice-IBP interface that align with the ice-lattice of nascent crystals and increase the energy required for expansion of the ice front. ${ }^{23,25,26}$ While the 
exact mechanism is still up for debate, genetic mutation studies have demonstrated that polar residues play a critical role. ${ }^{27-31}$

IBPs display ice recrystallization inhibition (IRI) activity, a measure of ice growth mitigation, in nanomolar concentrations..$^{23,24}$ However, IBPs are difficult to produce in large quantities ${ }^{32,33}$ and can lose function in non-physiological environments. In a previous study by the authors, an IBP from Shewanella frigidimarina demonstrated IRI activity in alkaline solutions $(\mathrm{pH} \leq 12.7)$ despite partial denaturation. ${ }^{34}$ However, IRI activity was lost at higher $\mathrm{pH}$ due to further denaturation and subsequent degradation. While promising, these results demonstrate limited applicability for native IBPs in applications such as cement paste and concrete, in which pore solution $\mathrm{pH}$ can exceed 13.5.35

Polymer mimics exhibit unique advantages over native IBPs. Synthetic polymers offer benefits, including increased stability in non-neutral solutions, production scalability, and tunability of pendant moieties, molecular weights, and architectures. Poly(vinyl alcohol) (PVA) has exhibited promising biomimetic IRI activity in neutral and $\mathrm{pH} 13$ solutions. ${ }^{36-38}$ One study demonstrated that $27 \mathrm{kDa}$ PVA displays moderate IRI behavior at $1.6 \mu \mathrm{M},{ }^{39}$ which approaches the nanomolar efficacy of IBPs. However, one drawback of PVA is its potential to cryogelate, namely to form a physically cross-linked polymer network around ice crystals during freeze-thaw cycling. ${ }^{40,41}$ Cryogelation may limit the applicability of PVA as molecules must be present in solution for ice growth inhibition. ${ }^{42}$

We posit that understanding the contribution of pendent functional groups found on residues responsible for ice-binding in IBPs would facilitate the fabrication of a synthetic IBP mimic with enhanced IRI activity at nanomolar concentrations. Taking inspiration from native IBPs, we first determined which ice-binding polypeptide residue exhibited the best ice growth inhibition in 
neutral phosphate-buffered saline (PBS). We determined that the polypeptide, poly(threonine) $(p T h r)$, demonstrated the highest IRI activity in PBS. Threonine is a common residue found on the ice-binding face of IBPs, ${ }^{30,31}$ and our work elucidated that it is able to elicit IRI activity as a homopolypeptide even without the lattice matching provided by protein structure, demonstrating a novel contribution for determining a potent pendant moiety for synthetic mimics. Using $p T h r$ as a molecular model, we synthesized a molecular weight sweep of a bioinspired synthetic mimic, poly(2-hydroxypropyl methacrylamide) ( $p H P M A)$, and investigated potential for IRI activity in PBS and in alkaline PBS solutions to determine potency and resiliency of IRI activity at neutral and elevated $\mathrm{pH}$. We determined that $\mathrm{pHPMA}$ demonstrated IRI activity at low molecular weights and nanomolar concentrations, thereby rivaling the potency of native IBPs and outperforming PVA, the leading synthetic in the field. Additionally, $p H P M A$ retained potent IRI activity in $\mathrm{pH}$ 13 PBS solution, demonstrating a unique advantage over native IBPs with increased resilience for applications in chemically aggressive non-physiological environments. Our results substantiate that $p H P M A$ offers a revolutionary bioinspired synthetic solution for mitigating ice crystal growth for a myriad of materials science and engineering applications.

\section{Materials and Methods}

Materials. 1-threonine (Thr), 1-arginine (Arg), l-glutamic acid (Glu), $7.6 \mathrm{kDa}$ poly(1-threonine) (pThr), $15 \mathrm{kDa}$ poly(l-arginine) (pArg), $12.1 \mathrm{kDa}$ poly(l-glutamic acid) ( $p G l u)$, and $45 \mathrm{kDa}$ poly(2hydroxypropyl methacrylamide) ( $p H P M A)$ were purchased from Sigma Aldrich to test for ice interactions without further purification. Molecular weights for homo-polypeptides and $45 \mathrm{kDa}$ pHPMA were provided by Sigma Aldrich (Supplementary Table 1). The authors synthesized other molecular weights of $p H P M A$ in-house. Phosphate buffered saline (PBS) was purchased from 
Fisher Scientific without further modification. $\mathrm{NaOH}$ was utilized to create PBS solutions with a $\mathrm{pH}$ of $8,10,12$, or 13. All other materials were purchased through Sigma Aldrich and used without further purification unless otherwise mentioned. Methacryloyl chloride was vacuum distilled twice and stored in a freezer $\left(-20^{\circ} \mathrm{C}\right)$ under nitrogen until use. All syntheses and polymerizations were performed under nitrogen atmosphere at ambient temperature. Nuclear magnetic resonance spectroscopy (NMR) experiments were performed on a Bruker AVIII (US) $400 \mathrm{MHz}$ spectrometer (FT $\left.400 \mathrm{MHz}{ }^{1} \mathrm{H}\right)$. Chemical shifts were referenced to an internal solvent signal and are reported in parts per million relative to tetramethyl silane. Polymer molecular weights were calculated utilizing monomer conversion through quantitative ${ }^{1} \mathrm{H}$ NMR relative to dimethylformamide internal standard.

Determination of a molecular model. Peptides (Thr, Arg, Glu) and homo-polypeptides ( $p$ Thr, $p A r g, p G l u)$ were loaded into PBS at a concentration of $10 \mathrm{mg} / \mathrm{mL}$ unless otherwise stated. Glu had a maximum concentration of $8.6 \mathrm{mg} / \mathrm{mL}$ due to solubility limitations and was compared against $10 \mathrm{mg} / \mathrm{mL}$ concentrations for other peptides. Figure 1 shows the chemical structure of the peptides and homo-polypeptides used in this study. The pendant functional group for peptides chosen for this study were inspired by the ice-binding residues of IBPs, which typically exhibit polar structures with the ability to interact with polar water and ice. For this study, a neutral polar, cationic polar, and anionic polar structure were chosen to determine the best pendant functionality for ice interaction in neutral solution. The material with the best ice recrystallization inhibition activity in PBS was used to determine the molecular model for synthetic replication. 
L-threonine (Thr)

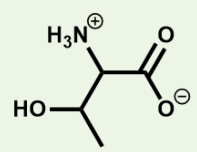

poly(L-threonine) ( $p T h r)$

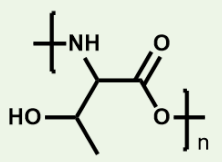

L-arginine (Arg)

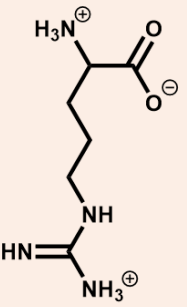

poly(L-arginine) ( $p A r g)$

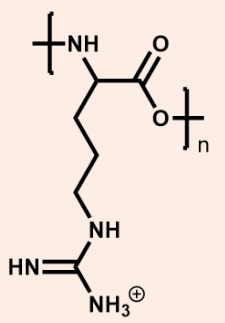

L-glutamic acid (G/u)

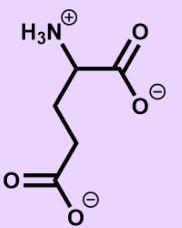

poly( $L$-glutamic acid) (pGlu)

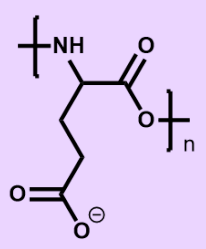

2-hydroxypropyl

methacrylamide (HPMA)

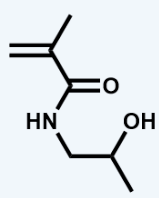

poly(2-hydroxypropyl methacrylamide) ( $p H P M A)$

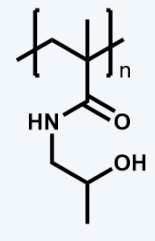

Figure 1. Chemical structure of molecules investigated in this study. Top row: peptides (Thr, Arg, Glu) and synthetic $T h r$ mimic 2-hydroxypropyl methacrylamide (HPMA). Bottom row: homopolypeptides (pThr, pArg, pGlu) and synthetic pThr mimic poly(2-hydroxypropyl methacrylamide) ( $p H P M A)$.

Ice Recrystallization Inhibition Activity. To determine ice recrystallization inhibition (IRI) activity, a splat assay was adapted from Knight et al. ${ }^{42}$ Briefly, peptides (Thr, Arg, Glu) and homopolypeptides (pThr, pArg, pGlu) were loaded in PBS at a concentration of $10 \mathrm{mg} / \mathrm{mL} .10 \mu \mathrm{L}$ samples were dispensed onto a microscope slide on top of an aluminum block chilled with dry ice from $1.7 \mathrm{~m}$ through a PVC pipe to obtain a monolayer of ice crystals. The slide was then transferred to an Otago nanoliter osmometer sample stage and annealed at $-4{ }^{\circ} \mathrm{C}$. The temperature was monitored using a bead-type thermocouple. Images were collected immediately after the splat was performed $\left(t_{0}\right)$ to verify polycrystalline ice formation and again at 30 minutes $\left(t_{30}\right)$ to observe ice recrystallization. Images were obtained using an Olympus BX41 microscope with an Olympus 
PLN 10X objective, equipped with an Axiocam 506 color camera on a 1" 1.0x 60N C-mount adapter. Splats were performed in triplicate. Similar to precedent research,,$^{34,42-44}$ IRI activity was determined through direct measurement of the mean largest grain size (MLGS) of ice crystals that formed in solution. ImageJ image processing software version 1.48v (National Institutes of Health, USA) was used to estimate the MLGS along the major axis. Data were collected by counting 10 of the largest grains from three different regions per splat to determine an average grain size $(n=$ 90) at $\mathrm{t}_{30}$ for each sample type. Based on IRI activity in PBS, $p T h r$ was determined to be the best performing homo-polypeptide, and the chemical structure for the threonine residue was used as a molecular model to choose a synthetic replicate, 2-hydroxypropyl methacrylamide (HPMA) and its respective polymer, $P H P M A$.

\section{Threonine Synthetic Mimics}

Synthesis of 2-hydroxypropyl methacrylamide. The molecule 2-hydroxypropyl methacrylamide (HPMA) and its respective polymer were chosen as a synthetic mimic for threonine, and their chemical structures can be seen in Figure 1. HPMA was chosen as a synthetic replicate for threonine because (a) the similarity of the pendant functional group (i.e., a hydroxyl adjacent to a methyl) and (b) the presence of an amide linkage. HPMA synthesis was performed by modifying a previously published protocol. ${ }^{45}$ Briefly, a flame-dried, two-neck round bottom flask with a stir bar was charged with anhydrous dichloromethane $(51 \mathrm{~mL}), \mathrm{Na}_{2} \mathrm{CO}_{3}(0.22$ moles, 1.1 eq.), and stirred to create a suspension. The reaction vessel was placed in an isopropanol:dry ice bath, and the solution temperature was lowered to $-10{ }^{\circ} \mathrm{C}$ prior to adding 2-aminopropanol $(0.20$ moles, 1 eq.). Distilled methacryloyl chloride $(0.20,1$ eq) was diluted by anhydrous dichloromethane (19 $\mathrm{mL}$ ) then added to the reaction solution dropwise over 30 minutes through a flame dried addition 
funnel during vigorous stirring. The temperature was maintained between $-10{ }^{\circ} \mathrm{C}$ to $-5{ }^{\circ} \mathrm{C}$ throughout the course of addition. The reaction was allowed to proceed as solution warmed to ambient temperature over $22 \mathrm{~h}$. The reaction solution was then centrifuged (2500 RPM, $5 \mathrm{~min}$ ), decanted, solids removed, then crystallized in dichloromethane at $-20^{\circ} \mathrm{C}$ overnight. Crystals were collected, dissolved in acetone, and then recrystallized at $-20{ }^{\circ} \mathrm{C}$ overnight. The $H P M A$ was collected as white crystals (8.6 g, 60 mmoles, $30 \%$ yield) and verified using ${ }^{1} \mathrm{H}$ NMR (Supplementary Figure 1).

Synthesis of poly(2-hydroxypropyl methacrylamide). To probe the potency of IRI activity of pHPMA, target molecular weights between $1.5 \mathrm{kDa}$ and $25 \mathrm{kDa}$ were synthesized using reversible addition-fragmentation chain transfer (RAFT) polymerization. Each RAFT polymerization proceeded as follows. In a nitrogen glove box, 4-cyano-4[(dodecylsulfanylthiocarbonyl)sulfanyl]pentanoic acid (CTA) and azobisisobutyronitrile (AIBN) were weighed out into a $20 \mathrm{~mL}$ scintillation vial, taken out of the glove box, and dissolved in an appropriate amount of HPLC grade methanol to fully dissolve the CTA and AIBN. HPMA (3.5 mmoles) was weighed and added directly to a storage flask with stir bar. The CTA/AIBN solution was transferred to the storage flask prior to sealing the flask under nitrogen and undergoing five freeze-pump-thaw cycles ( $<75$ millitorr vacuum). The flask was then sealed under nitrogen and placed in a $70{ }^{\circ} \mathrm{C}$ oil bath. The reaction was allowed to proceed for $16 \mathrm{~h}$ then quenched by placing in ice bath and exposing to air, prior to precipitating into acetone:diethyl ether $(2: 1)$. An offwhite/yellow semi-gelled solid was obtained. The sample was dissolved in $15 \mathrm{~mL}$ deionized $\mathrm{H}_{2} \mathrm{O}$, frozen with liquid nitrogen and placed on a lyophilizer (1.5 days) to yield a spongy offwhite/yellow solid. Lower molecular weight $(<5 \mathrm{kDa})$ samples underwent further purification post freeze-dry due to high solubility similarity of polymer in relation to the CTA and monomer. Freeze 
dried samples were suspended in acetone:ether and filtered (3 times) with increasing ether percentage $(5: 1,3: 1,1: 1)$. Solid off-white/yellow powder was collected. $p H P M A$ structure was verified using ${ }^{1} \mathrm{H}$ NMR (Supplementary Figure 2), and number average molecular weights were calculated (Supplementary Table 2). Polymer molecular weights and distributions were verified using SEC-MALS, and can be seen in Supplementary Figure 3. All pHPMA molecules are referred to by their weight average molecular weight as determined by SEC-MALS.

IRI activity. Thr, pThr, HPMA, and $45 \mathrm{kDa} p H P M A$ were loaded at $10 \mathrm{mg} / \mathrm{mL}$ into $\mathrm{pH}$ adjusted PBS solutions (PBS pH 8, PBS pH 10, PBS pH 12, PBS pH 13). To examine the potency of $p T h r$ and $45 \mathrm{kDa}$ PHPMA, IRI activity was determined after performing 10 -fold dilutions until IRI activity in PBS was no longer observed. $p H P M A$ with target molecular weights between $1 \mathrm{kDa}$ and $25 \mathrm{kDa}$ were synthesize and tested using 10-fold dilutions until IRI activity in PBS was no longer observed. IRI efficacy was determined using a modified splat assay, as described above.

\section{Results and Discussion}

Determining a molecular model. A molecular model for synthetic replicates was determined by investigating the IRI activity of three peptides inspired by ice-binding residues of IBPs (Thr, $A r g, G l u)$ and their homo-polypeptide counterparts ( $p T h r, p A r g, p G l u)$. The IRI activity, represented as percent mean largest grain size (MLGS) relative to PBS control solution for each peptide or homo-polypeptide at $10 \mathrm{mg} / \mathrm{mL}$ can be seen in Figure 2a, and representative micrographs at $\mathrm{t}_{30}$ are shown in Supplementary Figure 4. Peptides (Thr, Arg, Glu) and pArg did not exhibit noticeable IRI activity. Both $p T h r$ and $p G l u$ demonstrated IRI activity at $10 \mathrm{mg} / \mathrm{mL}$ in PBS solution, seen as a reduction of MLGS by $65.2 \%$ and 59.3\%, respectively. To determine if $p T h r$ or $p G l u$ would be a better model for a synthetic replicate, both $p T h r$ and $p G l u$ were tested for 
IRI activity using 10-fold dilutions (Figure 2b), and the homo-polypeptide that exhibited IRI at lower concentrations was taken as the better performing molecule. Representative micrographs at $\mathrm{t}_{30}$ for dilutions are shown in Supplementary Figure 5. In PBS, $p$ Thr exhibited significant IRI activity compared to PBS control solution at 1 and $0.1 \mathrm{mg} / \mathrm{mL}$ with reductions of $60.9 \%$ and $71.9 \%$, respectively. $p$ Glu did not inhibit ice growth at concentrations $\leq 1 \mathrm{mg} / \mathrm{mL}$, thus, $p T h r$ was taken as the molecular model to use for synthetic replication. Previous genetic mutation studies have shown that threonine is a common residue in IBPs to facilitate the organization of semi-liquid waters at the ice-binding surface,,$^{30,46}$ corroborating these results.
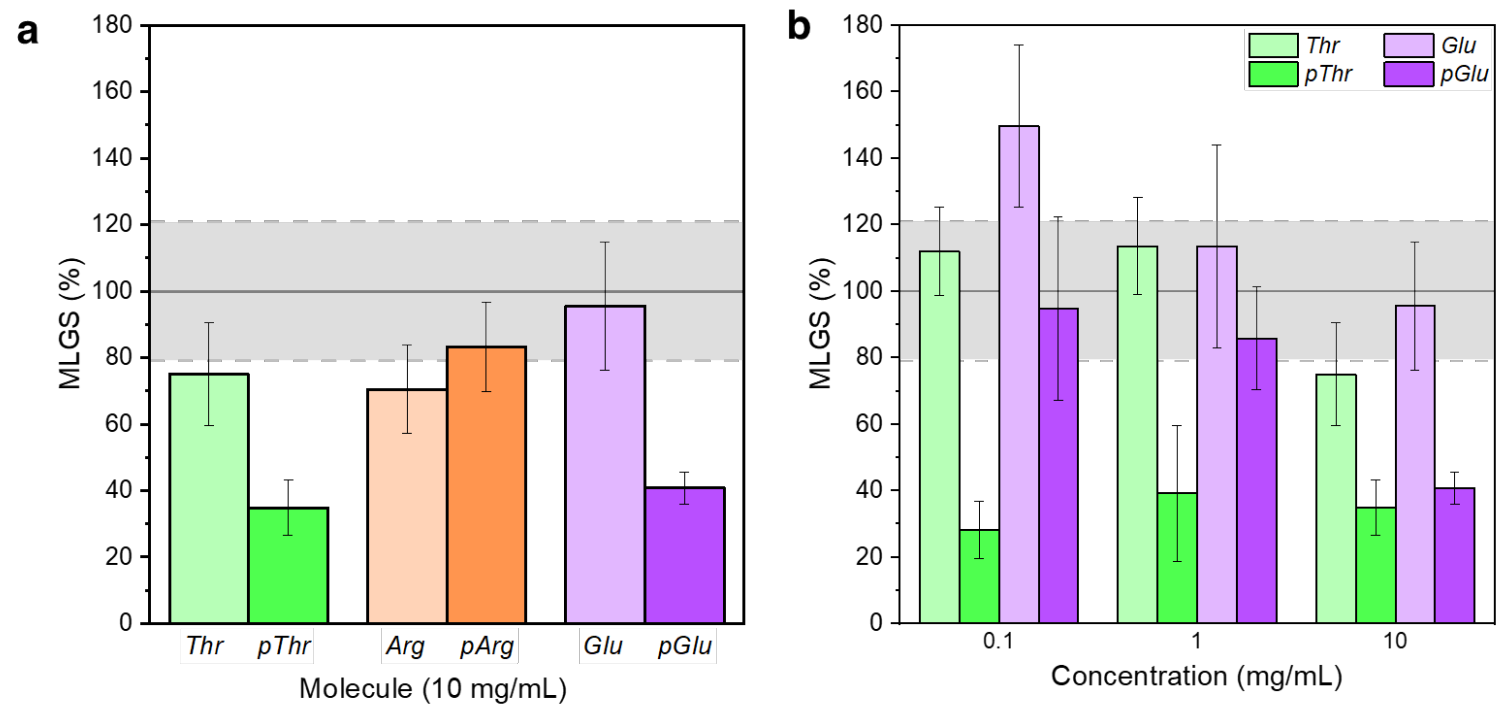

Figure 2. Ice recrystallization inhibition activity of peptides and polypeptides in PBS. a, Percent mean largest grain size (MLGS) relative to PBS control solution for Thr, pThr, Arg, pArg, Glu, and $p G l u$ at $10 \mathrm{mg} / \mathrm{mL}$ in PBS. b, Percent MLGS relative to PBS control solution for Thr, $p T h r$, $G l u$, and $p G l u$ at $0.1,1$, and $10 \mathrm{mg} / \mathrm{mL}$ in PBS. The gray band indicates the range of ice crystal MLGS for the PBS control solution. Error bars represent standard deviation for 90 ice grains across 3 samples. 
Ice Recrystallization Inhibition Activity.pThr and $45 \mathrm{kDa}$ pHPMA were tested for a minimum concentration needed to exhibit IRI activity. The relative activity compared to PBS control solution can be seen in Figure 3a. Thr and HPMA were also diluted and tested for IRI, however, as neither Thr nor HPMA exhibited IRI activity, values were not reported. The relative activity compared to the respective control solution, either PBS or alkaline PBS solutions, for Thr, $p T h r, H P M A$, and $45 \mathrm{kDa}$ PHPMA can be seen in Figure 3b and matches literature precedence for expected growth. ${ }^{47,48}$ Representative micrographs at $\mathrm{t}_{30}$ for splats performed can be seen in Supplementary Figures 6-8. Alkaline control solutions demonstrated varied crystal sizes at $t_{30}$ compared to PBS due to contributions of solution constituents that effect the recrystallization process ${ }^{49,50}$ However, as the estimated grain size of $T h r, p T h r, H P M A$, and $45 \mathrm{kDa} p H P M A$ was normalized to each respective alkaline control solution, the reported \%MLGS demonstrates the efficacy of each molecule to inhibit growth in the respective environment.

$45 \mathrm{kDa}$ pHPMA demonstrated a concentration limit for IRI activity an order of magnitude lower than $p T h r$, seen as a reduction of ice crystal size at $0.01 \mathrm{mg} / \mathrm{mL}$, whereas $p T h r$ exhibited an IRI activity threshold at $0.1 \mathrm{mg} / \mathrm{mL}$ in PBS (Figure 3a). Both $p T h r$ and $45 \mathrm{kDa} p H P M A$ exhibited IRI activity at $10 \mathrm{mg} / \mathrm{mL}$ in all PBS pH solutions. Notably, $p T h r$ and $45 \mathrm{kDa} p H P M A$ exhibited IRI activity in $\mathrm{pH} 13$ solution, seen as a 70.9\% and a $79.6 \%$ reduction in ice crystal size, respectively. In all environments, both $T h r$ and HPMA demonstrated an average ice crystal size smaller than control solutions; however, the reduction in ice crystal size was within error and Thr and HPMA cannot be stated to have significant IRI activity. A previous study by Congdon et al. demonstrated that IRI activity can be improved by ensuring IRI active moieties are proximal to each other, ${ }^{48}$ which corroborates the results seen herein where Thr and HPMA do not exhibit IRI activity, but their respective polymers do. pHPMA exhibits higher IRI activity than $p T h r$ in alkaline $\mathrm{pH}$ 
solutions and at lower concentrations in PBS. As it has been shown that addressing the source of ice expansion is beneficial to preventing freeze-thaw damage in highly alkaline concrete pore solution $(\mathrm{pH} \geq 13),{ }^{51}$ pHPMA would likely be a promising additive to cement paste or concrete to prevent freeze-thaw damage.
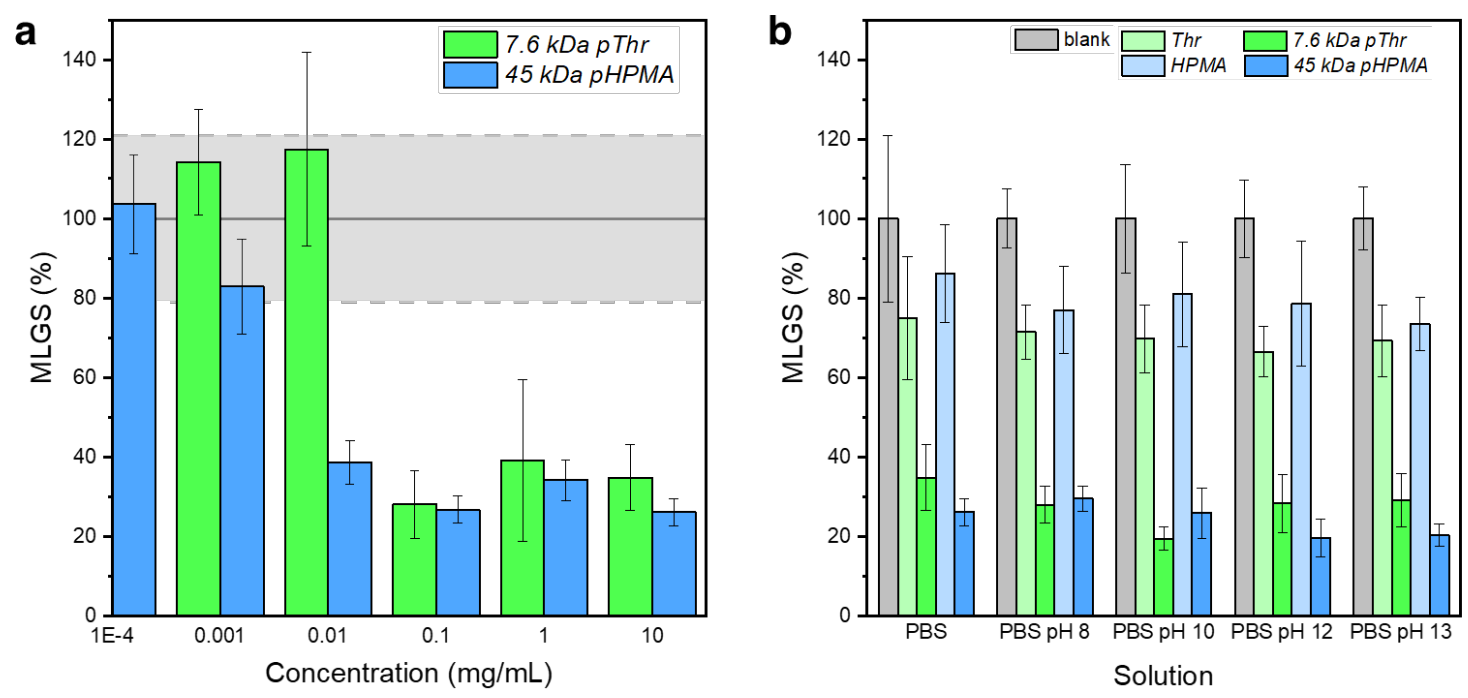

Figure 3. Ice recrystallization inhibition activity of $p T h r$ and synthetic mimic $p H P M A$. a, Percent MLGS relative to PBS control solution for $p T h r$ and $45 \mathrm{kDa} p H P M A$ at $0.0001,0.001,0.01,0.1$, 1 , and $10 \mathrm{mg} / \mathrm{mL}$. The gray band indicates the range of ice crystal mean largest grain size for the PBS control solution. b, MLGS relative to the respective control solution for Thr, pThr, HPMA, and $45 \mathrm{kDa}$ pHPMA at $10 \mathrm{mg} / \mathrm{mL}$ in: PBS and PBS alkaline $\mathrm{pH}$ solutions. Error bars represent standard deviation for 90 ice grains across 3 samples.

As the off-the-shelf $45 \mathrm{kDa} p H P M A$ exhibited potent IRI activity, the author's thought it pertinent to investigate the effect of molecular weight on the IRI activity of $p H P M A$. Previous 
studies have demonstrated that IRI activity varies with polymer molecular weight, with a lower molecular weight limit for polymers to demonstrate low to moderate IRI activity. ${ }^{36,38}$ Figure 4 demonstrates the effect of molecular weight and concentration on the IRI activity of $p H P M A$ compared to PBS control solution. Representative micrographs for splats at $t_{30}$ can be seen in

\section{Supplementary Figure 9.}

All molecular weights of pHPMA demonstrated IRI activity to concentrations of $0.01 \mathrm{mg} / \mathrm{mL}$ in PBS, seen as a reduction of MLGS between 50-80\%.32.8 kDa pHPMA demonstrated IRI activity down to $0.001 \mathrm{mg} / \mathrm{mL}$ in PBS, seen as a $40 \%$ reduction of MLGS compared to control solution. $0.001 \mathrm{mg} / \mathrm{mL} 32.8 \mathrm{kDa}$ pHPMA equates to $30 \mathrm{nM}$, demonstrating exceptional IRI activity similar to that of biological IBPs that exhibit IRI in nanomolar concentrations. ${ }^{23,24}$ 


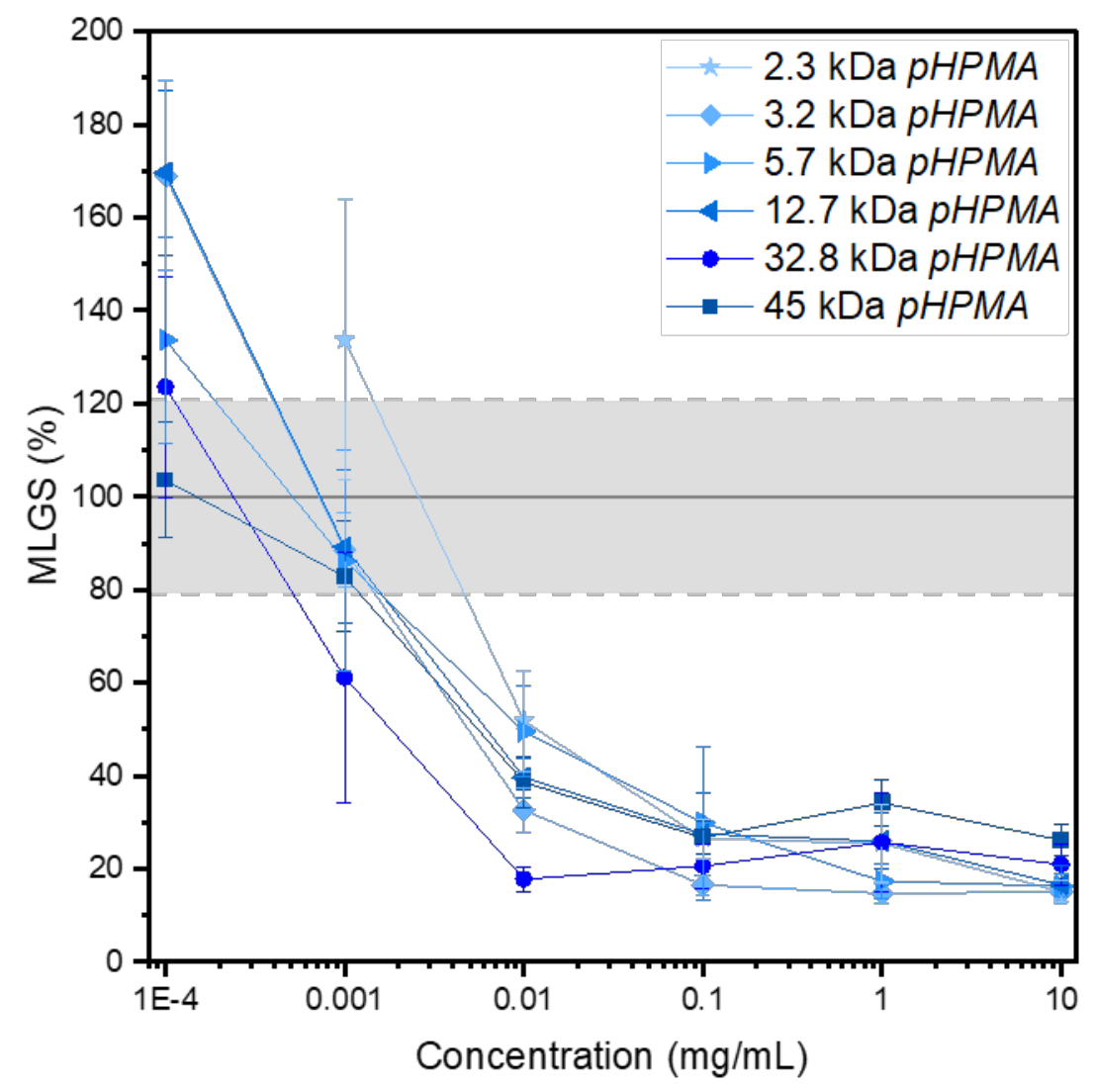

Figure 4. Influence of molecular weight on ice recrystallization inhibition activity of $p H P M A$. Percent MLGS relative to PBS control solution for $2.3 \mathrm{kDa} p H P M A, 3.2 \mathrm{kDa} p H P M A, 5.7 \mathrm{kDa}$ pHPMA, $12.7 \mathrm{kDa}$ pHPMA, $32.8 \mathrm{kDa}$ pHPMA, and $45 \mathrm{kDa} p H P M A$ at $0.0001,0.001,0.01,0.1,1$, and $10 \mathrm{mg} / \mathrm{mL}$ in PBS. The gray band indicates the range of ice crystal mean largest grain size for PBS control solution. Error bars represent standard deviation for 90 ice grains across 3 samples.

Previous studies that report on IBPs in $\mathrm{pH} 13$ solution found that the alkaline environment disrupted the proteins ability to prevent ice crystal growth, such as a previous study by the authors that demonstrated an IBP from the Antarctic bacterium Marinomonas primoryensis increased grain size in $\mathrm{pH} 13$ solution..$^{52}$ As the ice inhibition activity imparted by $p T h r$ and $45 \mathrm{kDa} p H P M A$ is not 
reliant on secondary structure like IBPs, they offer a distinct advantage at preventing ice growth in non-physiological environments compared to their biological counterparts. A previous study performed by Burkey et al. ${ }^{37}$ investigated the performance of PVA with a molecular weight of 27 $\mathrm{kDa}$ at $1 \mathrm{mg} / \mathrm{mL}$ in $\mathrm{pH} 13$ solution and found that the reduction in crystal size was $\sim 80 \%$, further corroborating that synthetics perform better in non-physiological environments than IBPs, offering potential ice inhibition strategies in engineering applications, such as freeze-thaw mitigation in cement and concrete in which the pore solution $\mathrm{pH}$ is $\geq 13$.

Other studies have looked at the effect of homo-polypeptides on ice recrystallization inhibition in neutral solutions. Graham et al. found similar results for a $16.2 \mathrm{kDa}$ poly(glutamic acid) and a $7.3 \mathrm{kDa}$ poly(lysine) at $5 \mathrm{mg} / \mathrm{mL}$ in PBS, which exhibited a $\sim 22 \%$ and a $\sim 21 \%$ reduction, respectively. In the study performed by Graham et al., the best performing molecule they tested was a $1.7 \mathrm{kDa}$ poly(d-proline), which exhibited a $\sim 42 \%$ reduction of ice crystal size at $10 \mathrm{mg} / \mathrm{mL}$ compared to the PBS control solution. ${ }^{53}$ A qualitative study performed by Knight et al. investigated homo-polypeptides inspired by IBP ice-binding residues and found the best performing was a 15 kDa poly-1-hydroxyproline, which exhibited 'a lot' of IRI activity from $0.1-1 \mathrm{mg} / \mathrm{mL}$ in saline, and 'some' at $0.01 \mathrm{mg} / \mathrm{mL} .{ }^{54}$ These literature results corroborate that $p H P M A$ outperforms the current synthetic mimics in the field at similar molecular weights and concentrations to provide a revolutionary bioinspired IRI active polymer.

PVA is a potent IRI active molecule with a pendant hydroxyl moiety on each repeat unit and currently remains one of the most potent and widely studied synthetics that displays IRI activity. An $8.6 \mathrm{kDa}$ PVA has been shown to exhibit a grain size reduction of $\sim 88 \%$ at $1 \mathrm{mg} / \mathrm{mL}$ and no reduction at $0.1 \mathrm{mg} / \mathrm{mL}$ respective to PBS control solution. ${ }^{36} \mathrm{pThr}$, which has a similar molecular weight of $7.6 \mathrm{kDa}$, comparatively exhibits a grain size reduction of $\sim 61 \%$ at $1 \mathrm{mg} / \mathrm{mL}$ and a $72 \%$ 
reduction at $0.1 \mathrm{mg} / \mathrm{mL}$ respective to $\mathrm{PBS}$ control solution, displaying higher activity than its PVA counterpart. Similarly, a $43 \mathrm{kDa}$ PVA exhibited a grain size reduction of $\sim 95 \%$ at $1 \mathrm{mg} / \mathrm{mL}, \sim 91 \%$ at $0.1 \mathrm{mg} / \mathrm{mL}$, and $\sim 19 \%$ at $0.01 \mathrm{mg} / \mathrm{mL}$ respective to the PBS control solution. ${ }^{36}$ Off-the-shelf pHPMA, which has a comparable molecular weight of $45 \mathrm{kDa}$, exhibited a grain size reduction of $\sim 66 \%$ at $1 \mathrm{mg} / \mathrm{mL}, \sim 73 \%$ at $0.1 \mathrm{mg} / \mathrm{mL}$, and $\sim 61 \%$ at $0.01 \mathrm{mg} / \mathrm{mL}$ respective to the PBS control solution. It was determined that all $p H P M A$ polymers outperformed PVA counterparts at similar molecular weights. For example, at $0.01 \mathrm{mg} / \mathrm{mL}$ in PBS, $32.8 \mathrm{kDa}$ pHPMA exhibited an $80 \%$ reduction of ice grain size, whereas $27 \mathrm{kDa}$ PVA demonstrated a $24 \%$ increase of ice grain size. ${ }^{37}$ Additionally, at $0.1 \mathrm{mg} / \mathrm{mL}$ in PBS, $2.3 \mathrm{kDa} p H P M A$ exhibited over twice the IRI activity as 1.5 $\mathrm{kDa}$ PVA, seen as a reduction in ice grain size of $74 \%$ compared to $30 \%$, respectively. A visual comparison between PHPMA to PVA of similar molecular weights can be seen in Figure 5, and these results lend to the hypothesis that the threonine pendant moiety, a hydroxyl adjacent to a methyl, may result in more potent IRI activity than PVA at similar molecular weights. IBPs have been shown to demonstrate IRI down to nanomolar concentrations, ${ }^{23,24}$ and $32.8 \mathrm{kDa} p H P M A$ exhibited IRI activity at $0.001 \mathrm{mg} / \mathrm{mL}(30 \mathrm{nM})$, demonstrating that $p H P M A$ has an IRI efficacy rivaling that of IBPs.

While PVA exhibits potent IRI activity at low concentrations and in elevated $\mathrm{pH}$ environments, it has been shown to cryogelate upon exposure to repetitive freeze-thaw cycling. ${ }^{40,41}$ In contrast, pHPMA has only been shown to crosslink in the presence of a chemical crosslinker. ${ }^{55,56}$ This creates a unique advantage for $p H P M A$ to supersede PVA as a novel, low-concentration additive to mitigate ice crystal growth. 


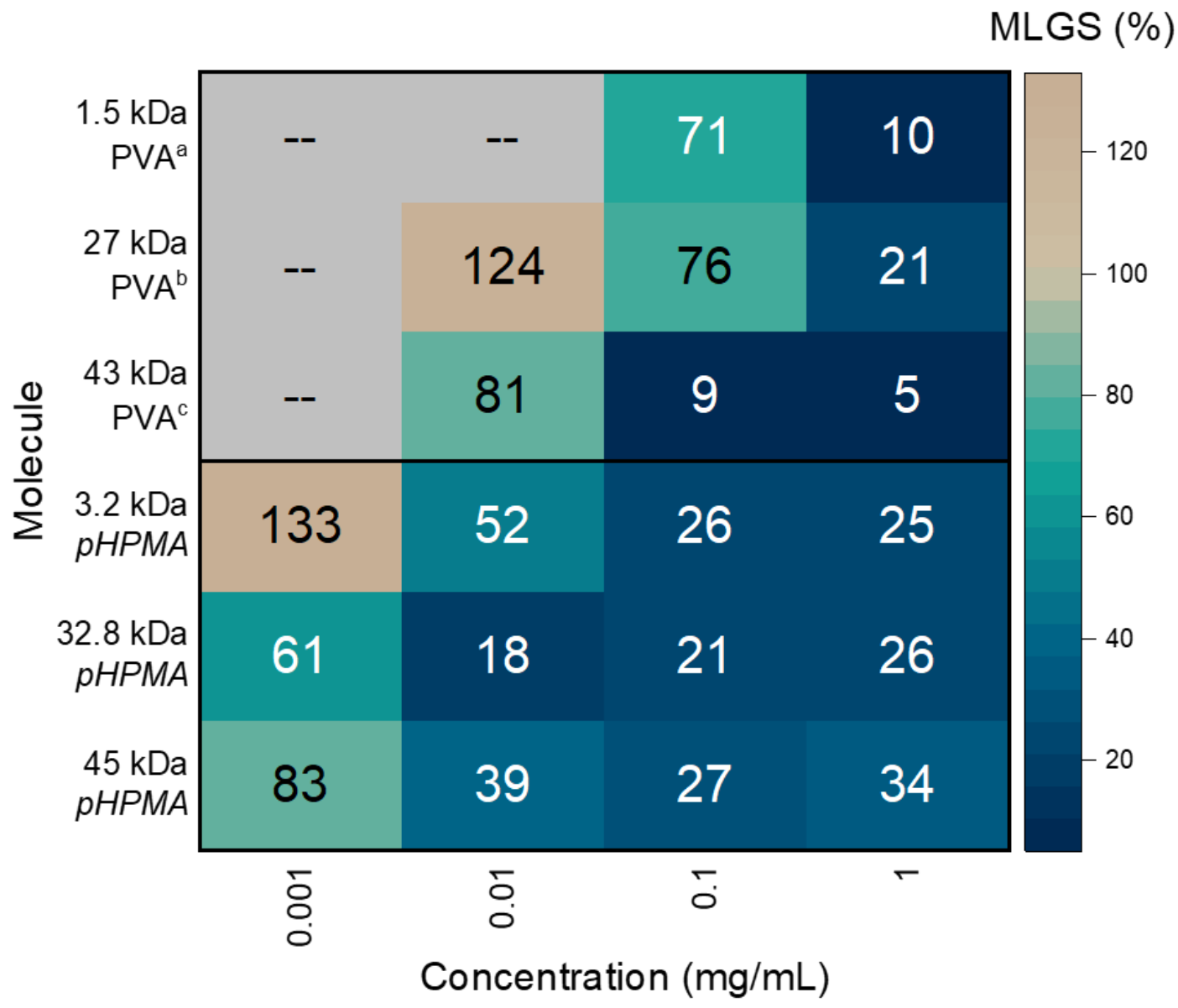

Figure 5. Comparison of ice recrystallization inhibition activity between PVA and $p H P M A$ in PBS. The IRI activity of synthesized and off-the-shelf $p H P M A$ was compared to previously studied PVA of similar molecular weights as a function of concentration, where darker squares indicate higher IRI activity. Values listed in each box represent mean largest grain size compared to control solution. ${ }^{\mathrm{a}}$ Data from Phillips et al. ${ }^{38}{ }^{\mathrm{b}}$ Data from Burkey et al. ${ }^{37}$ cData from Congdon et al.${ }^{36}$

\section{Conclusions}

This study examined the ability of three peptides (Thr, Arg, Glu) and their homo-polypeptides ( $p T h r, p A r g, p G l u$ ) to inhibit ice crystal growth in PBS. pThr exhibited the most promising IRI activity in PBS and was then used as a molecular model to choose an off-the-shelf synthetic 
replicate to test in alkaline environments. 2-hydroxypropyl methacrylamide (HPMA) and its respective polymer, $p H P M A$ were investigated as a synthetic mimic as they exhibit a pendant functional group similar to threonine. Thr, $p T h r, H P M A$, and $45 \mathrm{kDa} p H P M A$ were investigated for IRI activity. $45 \mathrm{kDa}$ pHPMA was shown to have IRI activity at ultra-low concentrations $(0.01$ $\mathrm{mg} / \mathrm{mL}$ ) in PBS and demonstrated an $\sim 80 \%$ reduction in ice crystal size in $\mathrm{pH} 13$ solution. After determining that off-the-shelf $p H P M A$ exhibited potent IRI activity, a molecular weight sweep was synthesized and investigated for a lower limit of IRI activity. It was found that $p H P M A$ with molecular weights as low as $2.3 \mathrm{kDa}$ exhibited potent IRI activity at concentrations $\leq 0.01 \mathrm{mg} / \mathrm{mL}$, demonstrating a novel new material with potent IRI activity akin to those of biological IBPs.

In conclusion, these results suggest that the peptide threonine (and polymers thereof) exhibits an important chemical structure to mimic in the design of biologically inspired macromolecules that exhibit ice interaction. Molecules based on its chemical structure, such as $p H P M A$, exhibit potent IRI activity in nanomolar concentrations. Additionally, peptide or synthetic polymers with pendant functional groups similar to threonine are promising potential additives for mitigating ice growth in both physiological and non-physiological environments.

\section{ASSOCIATED CONTENT}

Supporting Information. Supplementary Table 1 provides polymer properties as provided by Sigma Aldrich. Supplementary Figure 1 shows N-(2-hydroxypropyl)methacrylamide synthesis and verification. Supplementary Figure 2 shows poly[N-(2-hydroxypropyl)methacrylamide] synthesis verification. Supplementary Table 2 displays results of HPMA RAFT Polymerization. Supplementary Figure 3 provides SEC-MALS verification of $p H P M A$ molecular weights and distributions. Supplementary Figure 4 exhibits representative micrographs for the IRI splat assay 
for peptides and polypeptides. Supplementary Figure 5 provides representative micrographs for the concentration dependence of Thr, pThr, Glu, and $p G l u$ in PBS. Supplementary Figure 6 demonstrats representative micrographs for the concentration dependence of $7.6 \mathrm{kDa} p T h r$ and $45 \mathrm{kDa} p H P M A$ in PBS. Supplementary Figure 7 exhibits representative micrographs for the concentration dependence of Thr and HPMA in PBS. Supplementary Figure 8 displays representative micrographs for $T h r, 7.6 \mathrm{kDa} p T h r, H P M A$, and $45 \mathrm{kDa} p H P M A$ at $10 \mathrm{mg} / \mathrm{ml}$ in PBS at various $\mathrm{pH}$. Supplementary Figure 9 shows representative micrographs for the pHPMA molecular weight sweep IRI in PBS. Supplementary Figure 10 demonstrates dynamic ice shaping capabilities of $p T h r$ and synthetic mimic $p H P M A$.

The following files are available free of charge.

Polymer characterization and IRI micrographs (word doc)

\section{AUTHOR INFORMATION}

\section{Corresponding Author}

* Corresponding Author, T +1 303492 2621, F +1 303492 7317, E wsrubar@ colorado.edu

\section{Author Contributions}

The manuscript was written through contributions of all authors. All authors have given approval to the final version of the manuscript. Data curation, E.A.D., L.F.G., and A.J.L.; formal analysis, E.A.D., L.F.G., J.D.W., and A.J.L.; funding acquisition, G.M.M. and W.V.S.III; investigation, E.A.D., L.F.G., J.D.W., and A.J.L.; methodology, E.A.D., J.D.W., G.M.M., and W.V.S.III; supervision, G.M.M. and W.V.S.III; writing-original draft, E.A.D.; writing-review \& editing, 
L.F.G., J.D.W., A.J.L., G.M.M., and W.V.S.III. All authors have read and agreed to the published version of the manuscript.

\section{Funding Sources}

Financial support came from the United States (US) National Science Foundation (Award No. CMMI-1727788), the National Highway's Cooperative Research Program (NCHRP) (Award No. NCHRP-204), and the National Institutes of Health (Award No. R35GM119702).

\section{ACKNOWLEDGMENT}

This research was made possible by the Department of Civil, Environmental, and Architectural Engineering, the College of Engineering and Applied Sciences, and the Living Materials Lab at the University of Colorado Boulder with financial support from the United States (US) National Science Foundation (Award No. CMMI-1727788), the National Science Foundation Graduate Research Fellowship Program. This research was made possible by the Department of Chemistry at Colorado State University and the National Highway's Cooperative Research Program (NCHRP) (Award No. NCHRP-204), and the National Institutes of Health (Award No. R35GM119702). A special thanks to Jacob Lessard and the Sumerlin Research Group at the University of Florida for providing SEC-MALS verification. This work represents the views of the authors and not necessarily those of the sponsors.

\section{ABBREVIATIONS}

IBPs, ice-binding proteins; IRI, ice recrystallization inhibition; $p T h r$, poly(threonine); PBS, phosphate buffered saline; pHPMA, poly(2-hydroxypropyl methacrylamide); PVA, poly(vinyl alcohol); Thr, L-threonine; Arg, L-arginine; Glu, L-glutamic acid; $p A r g$, poly(L-arginine); $p G l u$, poly(L-glutamic acid); NMR, nuclear magnetic resonance; $H P M A$, 2-hydroxypropyl 
methacrylamide; MLGS, mean largest grain size; RAFT, reversible addition-fragmentation chain transfer; CTA, chain transfer agent; AIBN, azobisisobutyronitrile; SEC-MALS, size exclusion chromatography with multi-angle light scattering detector.

\section{REFERENCES}

1. Eisenberg, D. \& Kauzmann, W. The structure and properties of water. (Oxford University Press on Demand., 2005).

2. Voitkovskii, K. F. Translation of The Mechanical Properties of Ice. (1960).

3. Kasper, J. C. \& Friess, W. The freezing step in lyophilization: Physico-chemical fundamentals, freezing methods and consequences on process performance and quality attributes of biopharmaceuticals. Eur. J. Pharm. Biopharm. 78, 248-263 (2011).

4. Costantino, H. R. \& Pikal, M. J. Lyophilization of biopharmaceuticals. (Springer Science \& Business Media, 2004).

5. Harding, K. Genetic integrity of cryopreserved plant cells: A review. Cryo-Letters 25, 322 (2004).

6. John Morris, G. \& Acton, E. Controlled ice nucleation in cryopreservation - A review. Cryobiology 66, 85-92 (2013).

7. Kaleda, A., Tsanev, R., Klesment, T., Vilu, R. \& Laos, K. Ice cream structure modification by ice-binding proteins. Food Chem. 246, 164-171 (2018). 
8. Soukoulis, C. \& Fisk, I. Innovative ingredients and emerging technologies for controlling ice recrystallization, texture, and structure stability in frozen dairy desserts: A review. Critical Reviews in Food Science and Nutrition 56, 2543-2559 (2016).

9. Scherer, G. W. \& Valenza, J. J. Mechanisms of Frost Damage. Mater. Sci. Concr. 7, 209$246(2005)$.

10. Scherer, G. W. Crystallization in pores. Cem. Concr. Res. 29, 1347-1358 (1999).

11. Liu, L. et al. Analysis of damage development in cement paste due to ice nucleation at different temperatures. Cem. Concr. Compos. 53, 1-9 (2014).

12. Duman, J. G. \& Olsen, T. M. Thermal hysteresis protein activity in bacteria, fungi, and phylogenetically diverse plants. Cryobiology 30, 322-328 (1993).

13. Moffatt, B., Ewart, V. \& Eastman, A. Cold comfort: Plant antifreeze proteins. Physiol. Plant. 126, 5-16 (2006).

14. Hoshino, T. et al. Antifreeze proteins from snow mold fungi. Can. J. Bot. 81, 1175-1181 (2003).

15. Xiao, N. et al. Comparison of functional properties of two fungal antifreeze proteins from Antarctomyces psychrotrophicus and Typhula ishikariensis. FEBS J. 277, 394-403 (2010).

16. Marshall, C. B., Fletcher, G. L. \& Davies, P. L. Hyperactive antifreeze protein in a fish. Nature 429, 153 (2004).

17. DeVries, A. L. The role of antifreeze glycopeptides and peptides in the freezing avoidance of antarctic fishes. Comp. Biochem. Physiol. -- Part B Biochem. 90, 611-621 (1988). 
18. Davies, P. L., Hew, C. L. \& Fletcher, G. L. Fish antifreeze proteins: physiology and evolutionary biology. Can. J. Zool. 66, 2611-2617 (1988).

19. Liou, Y. C., Thibault, P., Walker, V. K., Davies, P. L. \& Graham, L. A. A complex family of highly heterogeneous and internally repetitive hyperactive antifreeze proteins from the beetle Tenebrio molitor. Biochemistry 38, 11415-11424 (1999).

20. Graham, L. A. \& Davies, P. L. Glycine-rich antifreeze proteins from snow fleas. Science (80-. ). 310, 461-461 (2005).

21. Vance, T. D. R. R., Graham, L. A. \& Davies, P. L. An ice-binding and tandem betasandwich domain-containing protein in Shewanella frigidimarina is a potential new type of ice adhesin. FEBS J. 285, 1511-1527 (2018).

22. Gilbert, J. A., Hill, P. J., Dodd, C. E. R. \& Laybourn-Parry, J. Demonstration of antifreeze protein activity in Antarctic lake bacteria. Microbiology 150, 171-180 (2004).

23. Dolev, M. B., Braslavsky, I. \& Davies, P. L. Ice-Binding Proteins and Their Function. Аnпи. Rev. Biochem. 85, 515-542 (2016).

24. Davies, P. L. Ice-binding proteins: A remarkable diversity of structures for stopping and starting ice growth. Trends Biochem. Sci. 39, 548-555 (2014).

25. Garnham, C. P., Campbell, R. L. \& Davies, P. L. Anchored clathrate waters bind antifreeze proteins to ice. Proc. Natl. Acad. Sci. U.S. A. 108, $7363-7367$ (2011). 
26. Chakraborty, S. \& Jana, B. Optimum Number of Anchored Clathrate Water and Its Instantaneous Fluctuations Dictate Ice Plane Recognition Specificities of Insect Antifreeze Protein. J. Phys. Chem. B 122, 3056-3067 (2018).

27. Zhang, D. Q., Liu, B., Feng, D. R., He, Y. M. \& Wang, J. F. Expression, purification, and antifreeze activity of carrot antifreeze protein and its mutants. Protein Expr. Purif. 35, 257-263 (2004).

28. Deluca, C. I., Davies, P. L., Ye, Q. \& Jia, Z. The Effects of Steric Mutations on the Structure of Type III Antifreeze Protein and its Interaction with Ice. J. Mol. Biol. 275, 515-525 (1998).

29. Baardsnes, J. et al. New ice-binding face for type I antifreeze protein. FEBS Lett. 463, 8791 (1999).

30. Haymet, A. D. J., Ward, L. G., Harding, M. M. \& Knight, C. A. Valine substituted winter flounder 'antifreeze': Preservation of ice growth hysteresis. FEBS Lett. 430, 301-306 (1998).

31. Baardsnes, J. \& Davies, P. L. Contribution of hydrophobic residues to ice binding by fish type III antifreeze protein. Biochim. Biophys. Acta - Proteins Proteomics 1601, 49-54 (2002).

32. Tab, M. M. et al. Large-Scale Production of Glaciozyma antarctica Antifreeze Protein 1 (Afp1) by Fed-Batch Fermentation of Pichia pastoris. Arab. J. Sci. Eng. 43, 133-141 (2018).

33. Tomalty, H. E., Graham, L. A., Eves, R., Gruneberg, A. K. \& Davies, P. L. LaboratoryScale Isolation of Insect Antifreeze Protein for Cryobiology. (2019). 
34. Delesky, E. A. et al. Ice-binding protein from Shewanella frigidimarinas inhibits ice crystal growth in highly alkaline solutions. Polymers (Basel). 11, 9-12 (2019).

35. Ghods, P., Isgor, O. B., McRae, G. \& Miller, T. The effect of concrete pore solution composition on the quality of passive oxide films on black steel reinforcement. Cem. Concr. Compos. 31, 2-11 (2009).

36. Congdon, T., Notman, R. \& Gibson, M. I. Antifreeze (Glyco)protein mimetic behavior of poly(vinyl alcohol): Detailed structure ice recrystallization inhibition activity study. Biomacromolecules 14, 1578-1586 (2013).

37. Burkey, A. A., Riley, C. L., Wang, L. K., Hatridge, T. A. \& Lynd, N. A. Understanding Poly(vinyl alcohol)-Mediated Ice Recrystallization Inhibition through Ice Adsorption Measurement and pH Effects. Biomacromolecules 19, 248-255 (2018).

38. Phillips, D. J., Congdon, T. R. \& Gibson, M. I. Activation of ice recrystallization inhibition activity of poly(vinyl alcohol) using a supramolecular trigger. Polym. Chem. (2016). doi:10.1039/C5PY01948F

39. Budke, C.\& Koop, T. Ice recrystallization inhibition and molecular recognition of ice faces by poly(vinyl alcohol). ChemPhysChem 7, 2601-2606 (2006).

40. Peppas, N. A. \& Stauffer, S. R. Reinforced uncrosslinked poly (vinyl alcohol) gels produced by cyclic freezing-thawing processes: a short review. J. Control. Release 16, 305-310 (1991).

41. Lozinsky, V. I. Cryotropic gelation of poly(vinyl alcohol) solutions. Usp. Khim. 67, 651655 (1998). 
42. Knight, C. A., Hallett, J. \& Devriess, A. L. Solute Effects on Ice Recrystallization: An Assessment Technique. Cryobiology 25, 55-60 (1988).

43. Czechura, P., Tam, R. Y., Dimitrijevic, E., Murphy, A. V.\& Ben, R. N. The importance of hydration for inhibiting ice recrystallization with C-linked antifreeze glycoproteins. J. Am. Chem. Soc. 130, 2928-2929 (2008).

44. Budke, C. et al. Quantitative efficacy classification of ice recrystallization inhibition agents. Cryst. Growth Des. 14, 4285-4294 (2014).

45. Ebbesen, M. F., Schaffert, D. H., Crowley, M. L., Oupický, D. \& Howard, K. A. Synthesis of click-reactive HPMA copolymers using RAFT polymerization for drug delivery applications. J. Polym. Sci. Part A Polym. Chem. 51, 5091-5099 (2013).

46. Jia, Z., DeLuca, C., Chao, H. \& Davies, P. L. Structural basis for the binding of a globular antifreeze protein to ice. Chemtracts 11, 229-232 (1998).

47. Graham, B., Fayter, A. E. R. R., Houston, J. E., Evans, R. C. \& Gibson, M. I. Facially Amphipathic Glycopolymers Inhibit Ice Recrystallization. J. Am. Chem. Soc. 140, 5682-5685 (2018).

48. Congdon, T. R., Notman, R. \& Gibson, M. I. Influence of Block Copolymerization on the Antifreeze Protein Mimetic Ice Recrystallization Inhibition Activity of Poly(vinyl alcohol). Biomacromolecules 17, 3033-3039 (2016).

49. Wu, S. et al. Ion-specific ice recrystallization provides a facile approach for the fabrication of porous materials. Nat. Commun. 8, 1-8 (2017). 
50. Duignan, T. T., Parsons, D. F. \& Ninham, B. W. Collins's rule, Hofmeister effects and ionic dispersion interactions. Chem. Phys. Lett. 608, 55-59 (2014).

51. Frazier, S. D. et al. Inhibiting Freeze-Thaw Damage in Cement Paste and Concrete by Mimicking Nature's Antifreeze. Cell Reports Phys. Sci. 1, 100060 (2020).

52. Delesky, E. A., Thomas, P. E., Charrier, M., Cameron, J. C. \& Srubar, W. V. Effect of pH on the activity of ice-binding protein from Marinomonas primoryensis. Extremophiles (2020). doi:10.1007/s00792-020-01206-9

53. Graham, B. et al. Polyproline as a Minimal Antifreeze Protein Mimic That Enhances the Cryopreservation of Cell Monolayers. Angew. Chemie - Int. Ed. 56, 15941-15944 (2017).

54. Knight, C. A., Wen, D. \& Laursen, R. A. Nonequilibrium antifreeze peptides and the recrystallization of ice. Cryobiology 32, 23-34 (1995).

55. Biancacci, I. et al. Optical imaging of the whole-body to cellular biodistribution of clinicalstage PEG-b-pHPMA-based core-crosslinked polymeric micelles. J. Control. Release 328, 805$816(2020)$.

56. Bryan, T., Getchell, S., Hill, M. \& Sumerlin, B. Facile Synthesis of Drug-Conjugated PHPMA Core- Crosslinked Star Polymers. 2Polymer Chem. 6, 4258-4263 (2015). 